\section{Kidney \\ Blood Pressure Research}

\title{
Renoprotective Effect of Angiotensin- Converting Enzyme Inhibitors and Angiotensin II Receptor Blockers in Diabetic Patients with Proteinuria
}

\author{
Feng-Yi Hsu ${ }^{a}$ Fang-Ju Lin ${ }^{a, b, c}$ Huang-Tz Ou ${ }^{d}$ Shih-Hui Huange \\ Chi-Chuan Wanga,c
}

aSchool of Pharmacy; ${ }^{b}$ Graduate Institute of Clinical Pharmacy, College of Medicine; 'Department of Pharmacy, National Taiwan University Hospital, Taipei; 'Institute of Clinical Pharmacy and Pharmaceutical Sciences, National Cheng Kung University, Tainan; eInternal Medicine, Penghu Hospital, Department of Health, The Executive Yua, Penghu, Taiwan

\section{Key Words}

Angiotensin II receptor blockers - Angiotensin-converting enzyme inhibitors - Comparative effectiveness - Diabetic nephropathy $\cdot$ Renoprotective effect

\begin{abstract}
Background/Aims: Limited evidence exists on the choice of angiotensin-converting enzyme inhibitors (ACEIs) and angiotensin II receptor blockers (ARBs) in diabetic patients with nephropathy. We aim to assess the renal effectiveness and safety of these drugs among diabetic nephropathy patients. Methods: This retrospective cohort study was conducted with diabetic nephropathy patients who initiated ACEI or ARB monotherapy. The primary outcome was a composite of end stage of renal disease and renal transplantation, and the secondary outcome was all-cause mortality. The safety endpoint was hyperkalemia. Results: Three thousand seven hundred and thirty-nine ACEI users and 3,316 ARB users were identified. ARBs seemed to be inferior to ACEIs given their poorer renal outcome (HR 1.31; 95\% CI, 1.15-1.50) and higher risk of hyperkalemia (HR 1.17; 95\% CI, 1.04-1.32). Among the four ACEIs compared, captopril was an inferior treatment choice given its poorer renal outcomes (HR 1.42; $95 \% \mathrm{CI}$, 1.05-1.93) and higher mortality rate (HR 1.25; 95\% CI, 1.01-1.55). Irbesartan appeared to be a poorer treatment choice among the three ARBs compared, given its inferior renal protective effect (HR 1.35; 95\% CI, 1.03-1.78). Conclusions: Our findings suggest ACEIs as a relatively more renoprotective and safer treatment as compared to ARBs. Captopril and irbesartan may be inferior to the other ACEIs and ARBs respectively.
\end{abstract}




\section{Kidney Blood Pressure Research}

\section{Introduction}

Diabetic nephropathy is a common complication among patients with diabetes mellitus (DM) and the leading cause of chronic kidney disease (CKD) in developed countries [1]. It involves an increase in proteinuria and decrease in glomerular filtration rate. The continuous kidney damage can lead to irreversible renal failure (i.e., end-stage renal disease; ESRD), which may eventually require dialysis or a kidney transplant.

Blood pressure control is essential to avoid the progression from uncomplicated diabetes to diabetic nephropathy [2], and angiotensin-converting enzyme inhibitors (ACEIs) and angiotensin II receptor blockers (ARBs) are two classes of agents that could effectively reduce the incidence of end-stage kidney disease [3, 4]. The current treatment guidelines recommend ACEI or ARB monotherapy for blood pressure control among non-pregnant diabetic patients with kidney disease [5]. The combination therapy of ACEIs and ARBs, however, is not recommended since it does not provide any additional clinical benefits and may increase the risk of hyperkalemia [6,7].

While current guidelines suggest ACEIs or ARBs for diabetic patients with modestly elevated urinary albumin excretion (30 - $299 \mathrm{mg}$ /day) and those with severe urinary albumin excretion ( $>300 \mathrm{mg} /$ day), it does not provide much detail in the choice of these drugs given the limited evidence [5]. Given that there are many ACEIs and ARBs available on the market, the clinical treatment of diabetic nephropathy becomes widely diverse and complex. As ACEI and ARB medications have different pharmacokinetic and pharmacodynamic profiles, it is important to differentiate and prioritize the use of various ACEIs and ARBs. Although recent research has studied the renoprotective effect of ACEI and ARB monotherapy in hypertensive patients with CKD or with DM, the renoprotective effects and safety of these therapies regarding thee diabetic nephropathy population remains undetermined $[8,9]$. Therefore, further investigation is necessary to examine the renal effectiveness and safety of ACEIs and ARBs in diabetic patients complicated with proteinuria.

The aims of this study were as follows: 1) compare the effectiveness and safety between ACEIs and ARBs, which are the first-line medication therapies for diabetic patients with proteinuria, 2) assess the effectiveness and safety of the most commonly used ACEIs and ARBs drugs, with individual drug comparisons within each class, and 3) evaluate the effectiveness and safety of ACEIs and ARBs among patients aged 65 or older since the elderly generally have a different pattern of disease and response to medications [10-13].

\section{Material and Methods}

\section{Data Source}

Data of the present study were obtained from the National Health Insurance Research Database (NHIRD), which contains data taken from the National Health Insurance (NHI) program that covers over 99\% of the entire 23 million residents in Taiwan since 1995 [14]. The NHIRD consists of information on beneficiaries' health care utilization, prescription drug use, and enrollment status. The present study used the Longitudinal Health Insurance Database (LHID), a sub-dataset of NHIRD, containing healthcare claims for a cohort of 1 million random samples from the beneficiaries enrolled in the NHI in 2000, 2005 and 2010 respectively. Therefore, the original database contains approximately 3 million people for analysis.

\section{Study Design and Sample}

We selected individuals who had initiated monotherapy with an ACEI or ARB from July 1, 2002 through December 31, 2013 and who had also been diagnosed with diabetic nephropathy (International Classification of Diseases, Ninth Revision, Clinical Modification [ICD-9-CM]: 250.40) or with both diabetes and proteinuria (ICD-9-CM: 791.0) at the same visit within 180 days before the first observed ACEI or ARB prescription. Patients were considered as having diabetes if they met one of the following criteria [15]: 


\section{Kidney Blood Pressure Research}

1. At least one inpatient diagnosis of diabetes (ICD-9-CM: 250)

2. Two or more outpatient diagnoses of diabetes

3. One outpatient diagnosis of diabetes and at least one outpatient prescription of antidiabetic medication at the same visit

We then identified patients who had not received a prescription of ACEIs or ARBs for at least 180 days before the first observed prescription during July 1, 2002 to December 31, 2013 (i.e., patients who had experienced a washout period), in order to select the new users of these drugs. To identify stable users, patients were required to have a second prescription of the same ACEI or ARB medication within 100 days following the first observed ACEI or ARB prescription. The second prescription date of the ACEI or ARB then served as the index date.

We excluded patients younger than 20 years old at the first prescription date, those who had more than 12 dialysis treatments within 180 days before the first prescription date, or who had received a catastrophic illness certificate for CKD with long-term dialysis before the first prescription date [16, 17]. We also excluded the renal transplant recipients (ICD-9-CM: 55.6, V42.0; procedure code: 76020B) at any time before the first prescription date. The study was reviewed and approved through an ethical review by the Research Ethics Committee of the National Taiwan University Hospital (NTUH-REC No. 201601003W).

\section{Treatment exposure}

Two exposure groups were included in this study: ACEI and ARB users. For the comparisons of individual ACEIs and ARBs, enalapril and valsartan were set as the reference agents since they are the most commonly used ACEI and ARB, respectively, from 2004 to 2014 [18]. Medications were identified according to the Anatomic Therapeutic Chemical (ATC) code, which is an internationally accepted classification system for drugs coordinated by the World Health Organization Collaborating Center for Drug Statistics Methodology [19].

Both intention-to-treat (ITT) and as-treated (AT) analyses were performed. In the ITT analysis, we analyzed all the patients based on their initial ACEI or ARB exposure, disregarding any subsequent change or discontinuance of these two types of treatments. On the other hand, the AT analysis took into consideration the subsequent changes in the exposure status and only evaluates the effectiveness and safety of the index ACEI or ARB medication during the time when patients consistently were exposed to them. Patients were censored if they switched or added a second blood pressure-lowering agent or discontinued their index exposure (defined with a 90-day gap).

\section{Dependent Variable}

Two effectiveness outcomes were assessed: 1) a composite outcome of ESRD and renal transplantation and 2) death. ESRD was confirmed based on the catastrophic illness certificate. All-cause mortality was considered as a secondary outcome defined as follows [20]:

1. withdrawal from the NHI program within 30 days after discharge from the hospital

2. having a death record in the catastrophic illness certificate file

3. having "death" as the transaction code in the inpatient file of NHI

The safety outcome, hyperkalemia, was defined by a diagnosis code of hyperkalemia (ICD-9-CM: 276.7) or the use of sodium or calcium polystyrene sulfonate (ATC code: V03AE01) during the follow-up period. Only patients who had received a sodium or calcium polystyrene sulfonate prescription once within a 3-month period were considered as having undergone a defined hyperkalemia event, in order to exclude the prophylactic treatment of hyperkalemia. Additionally, we performed a sensitivity analysis, which used "having a diagnosis code of hyperkalemia or receiving sodium or calcium polystyrene sulfonate at least once" as the definition of hyperkalemia. All patients were followed from the index date to the first event date, or to the end of this study, December 31,2013, whichever came first.

\section{Covariates}

Covariates included in this study were: age at the first prescription date, sex, geographic location, comorbidities, and other medications used. Comorbidities and prior medication use were measured during the 180 days before the first prescription date. Comorbidities and medications used included hypertension (ICD-9-CM: 401-405), hyperlipidemia (ICD-9-CM: 272), kidney disease (ICD-9-CM: 016.0, 042, 095.4, 189, 


\section{Kidney Blood Pressure Research}

223, 236.9, 250.4, 271.4, 274.1, 403-404, 440.1, 442.1, 446.21, 447.3, 572.4, 580-589, 590-591, 593, 642.1, 642.2, 753, 984) [8], stroke (ICD-9-CM: 434.91), coronary heart disease (ICD-9-CM: 410-414), neoplasms (ICD-9-CM: 140-239), drugs associated with nephrotoxicity [21, 22], antidiabetic medications, and other blood pressure-lowering agents [23].

\section{Statistical Analysis}

Propensity score weighting (standardized mortality/ morbidity ratio [SMR] weighting) was used to balance the baseline covariates. The balance of demographic and clinical characteristics between new ACEI and ARB users were examined based on the absolute standardized mean difference. A value of absolute standardized mean difference less than 0.1 indicated a negligible difference in potential confounders between the two study groups [24, 25].

Poisson regression model was used to calculate the incidence rate (per 100 patient-years) and the corresponding 95\% confidence intervals (CI). The adjusted results were expressed as hazard ratios (HRs) with 95\% CI derived from Cox proportional hazards models. All $p$-values were 2 -sided, and the significance level was set at 0.05 a priori. All analyses were conducted using SAS 9.4 (SAS Institute Inc., Cary, NC, USA).

\section{Results}

There were 509,277 patients who had received ACEI or ARB without combination therapy from July 01, 2012 to December 31, 2013 in the three LHID cohorts. After applying the inclusion and exclusion criteria, we combined the identified subjects from the three LHID cohorts, and ruled out 268 duplicate patients. After this, 7,055 patients remained for the present study, including 3,739 ACEI users and 3,316 ARB users. Given that some of the ACEIs or ARBs were less frequently prescribed and thus had a limited number of users, we only selected four commonly used ACEIs: captopril, enalapril, fosinopril and perindopril, and three common ARBs: irbesartan, losartan and valsartan, for the subgroup analyses.

Before propensity score weighting, the ARB users had larger proportion of hypertension, kidney disease and higher medication use rate, such as insulin, calcium channel blockers and thiazides, than ACEI users (Table 1). After propensity score weighting, all covariates between ACEI and ARB users were balanced (all absolute standardized mean differences were less than 0.1).

When comparing ACEIs and ARBs in the ITT analysis, our study indicated that ARB users had a higher risk of the primary composite renal outcome (HR: 1.31, 95\% CI: 1.15-1.50), and a greater rate of progress toward ESRD (HR: 1.30, 95\% CI: 1.13-1.49) as compared with ACEI users (Table 2). Additionally, although the results were not significant in the AT analysis, the same trend for higher rate of primary composite renal outcome in ARB users was still found. All-cause mortality was not significantly different between ACEI and ARB users in both ITT and AT analysis. Regarding the safety outcome, ARB users had a higher risk of hyperkalemia in the ITT analysis (HR: 1.17, 95\% CI: 1.04-1.32).

In the head-to-head comparisons of individual ACEIs, captopril users had a greater rate of primary composite renal outcome (HR: 1.42, 95\% CI: 1.05-1.93), a higher risk of progression toward ESRD (HR: 1.39, 95\% CI: 1.03-1.89), and a greater mortality rate in the ITT analysis (HR: 1.25 , 95\% CI: 1.01-1.55). No significant differences were found in the comparisons of fosinopril and perindopril versus enalapril. As for hyperkalemia, there were no significant difference between enalapril and any of the selected ACEI drugs (Table 3).

When comparing irbesartan and losartan to valsartan, we found that irbesartan was associated with a greater rate of primary composite renal outcome (HR: 1.35, 95\% CI: 1.031.78), and a higher risk of ESRD (HR: 1.34, 95\% CI: 1.01-1.77). No significant differences were observed in death and hyperkalemia between valsartan and all the selected ARBs (Table 4).

Lastly, we conducted a pre-specified subgroup analysis in targeted patients aged 65 years or older. Similar to the aforementioned findings for all adults, as compared to ACEI users, ARB users had a greater rate of primary composite renal outcome (HR: 1.84, 95\% 


\section{Kidney Blood Pressure Research}

Table 1. Baseline Characteristics of Diabetic Patients with Proteinuria Receiving ACEIs and ARBs

CI: 1.44-2.33), a higher risk of ESRD (HR: 1.77, 95\% CI, 1.39-2.26) and renal transplant events (HR: 5.14, 95\% CI: 1.12-23.61), and a relatively high risk of hyperkalemia (HR: 1.25, 95\% CI: 1.05-1.49) in the ITT analysis (Table 5). The same trend for higher rate of primary composite renal outcome was found in the AT analysis, but the results were not significant. In the analysis of individual ACEIs, captopril users had a greater rate of primary composite renal

Table 2. Incidence Rates and Hazard Ratios for Effectiveness and Safety Outcomes among ACEI and ARB Users
Kidney Blood Press Res 2017;42:358-368

\begin{tabular}{|l|l|}
\hline DOI: $10.1159 / 000477946$ & (C) 2017 The Author(s). Published by S. Karger AG, Base
\end{tabular} Published online: June 13, $2017 \quad$ www.karger.com/kbr

Hsu et al.: Renoprotective Effect of ACEIs and ARBs

\begin{tabular}{|c|c|c|c|}
\hline & \multicolumn{3}{|c|}{ Propensity-Score Weighting } \\
\hline & \multicolumn{2}{|c|}{ Before } & \multirow{2}{*}{$\begin{array}{c}\text { After } \\
\text { ACEI Users }\end{array}$} \\
\hline & ACEI Users & ARB Users & \\
\hline Characteristic n (\%) & $(n=3739)$ & $(n=3316)$ & $(n=3329) \%$ \\
\hline Age, mean (SD), y & $59.43(12.76)$ & $60.19(12.93)$ & $60.20(12.00)$ \\
\hline \multicolumn{4}{|l|}{ Age group, $\mathrm{y}$} \\
\hline $20-34$ & $123(3.29)$ & $97(2.93)$ & 2.80 \\
\hline $35-49$ & $674(18.03)$ & $540(16.28)$ & 16.26 \\
\hline $50-64$ & $1596(42.69)$ & $1453(43.82)$ & 43.55 \\
\hline$\geq 65$ & $1346(36.00)$ & $1226(36.97)$ & 37.39 \\
\hline Men sex & $2099(56.14)$ & $1881(56.72)$ & 56.90 \\
\hline \multicolumn{4}{|l|}{ Geographic location } \\
\hline Northern & $1719(45.97)$ & $1516(45.72)$ & 46.17 \\
\hline Middle & $951(25.43)$ & $776(23.40)$ & 23.68 \\
\hline Southern & $972(26.00)$ & $914(27.56)$ & 26.93 \\
\hline Eastern and other islands & $97(2.59)$ & $110(3.32)$ & 3.21 \\
\hline \multicolumn{4}{|c|}{ Jomorbid conditions within 180 days before the first prescription date } \\
\hline Coronary heart disease & $420(11.23)$ & $369(11.13)$ & 11.57 \\
\hline Heart failure & $91(2.43)$ & $90(2.71)$ & 2.89 \\
\hline Hyperlipidemia & $1521(40.68)$ & $1461(44.06)$ & 43.67 \\
\hline Hypertension & $1385(37.04)$ & $1547(46.65)$ & 47.31 \\
\hline Kidney disease & $1893(50.63)$ & $1985(59.86)$ & 60.07 \\
\hline Neoplasms & $249(6.66)$ & $223(6.72)$ & 6.51 \\
\hline Stroke & $115(3.08)$ & $126(3.80)$ & 4.03 \\
\hline \multicolumn{4}{|c|}{ Medication used within 180 days before the first prescription date } \\
\hline \multicolumn{4}{|l|}{ Diabetic medications } \\
\hline Insulin & $712(19.04)$ & $787(23.73)$ & 24.58 \\
\hline Oral hypoglycemic agents & $3083(82.46)$ & $2729(82.30)$ & 82.52 \\
\hline \multicolumn{4}{|l|}{ Blood pressure-lowering agents } \\
\hline Calcium channel blockers & $1053(28.16)$ & $1170(35.28)$ & 35.85 \\
\hline Loop diuretics & $266(7.11)$ & $282(8.50)$ & 8.41 \\
\hline Potassium sparing diuretics & $109(2.92)$ & $86(2.59)$ & 2.51 \\
\hline Thiazides & $435(11.63)$ & $513(15.47)$ & 15.32 \\
\hline $\begin{array}{l}\text { ACEIs and Other blood pressure- } \\
\text { lowering agents in combination }\end{array}$ & $14(0.37)$ & $53(1.60)$ & 2.20 \\
\hline $\begin{array}{l}\text { ARBs and Other blood pressure- } \\
\text { lowering agents in combination }\end{array}$ & $72(1.93)$ & $286(8.62)$ & 9.04 \\
\hline $\begin{array}{l}\text { Potassium sparing diuretics and } \\
\text { other diuretics in combination }\end{array}$ & $104(2.78)$ & $86(2.59)$ & 2.65 \\
\hline
\end{tabular}

$\nabla$

\begin{tabular}{|c|c|c|c|c|}
\hline & \multicolumn{2}{|c|}{$\begin{array}{c}\text { Incidence Rate per } 100 \text { Patient-years } \\
(95 \% \mathrm{CI}) \\
\end{array}$} & \multicolumn{2}{|c|}{ Hazard Ratio $(95 \% \mathrm{CI}), p$-Value } \\
\hline & ACEI Users & ARB Users & ACEI Users & ARB Users \\
\hline \multicolumn{5}{|l|}{ ITT } \\
\hline \multicolumn{5}{|l|}{ Effectiveness } \\
\hline Primary composite outcomes & $2.10(2.08-2.12)$ & $2.75(2.73-2.78)$ & 1 (Reference) & $1.31(1.15-1.50),<0.01^{*}$ \\
\hline ESRD & $2.06(2.04-2.08)$ & $2.67(2.64-2.69)$ & 1 (Reference) & $1.30(1.13-1.49),<0.01^{*}$ \\
\hline Renal transplant & $0.04(0.04-0.04)$ & $0.08(0.08-0.09)$ & 1 (Reference) & $1.94(0.80-4.66), 0.14$ \\
\hline \multicolumn{5}{|l|}{ Secondary outcome } \\
\hline Death & $3.54(3.51-3.56)$ & $3.68(3.65-3.71)$ & 1 (Reference) & $1.10(0.98-1.23), 0.10$ \\
\hline \multicolumn{5}{|l|}{ Safety } \\
\hline Hyperkalemia & $2.75(2.73-2.78)$ & $3.32(3.29-3.35)$ & 1 (Reference) & 1.17 (1.04-1.32), 0.01* \\
\hline \multicolumn{5}{|l|}{ AT } \\
\hline \multicolumn{5}{|l|}{ Effectiveness } \\
\hline Primary composite outcomes & $0.32(0.30-0.35)$ & $0.63(0.60-0.66)$ & 1 (Reference) & 2.17 (0.83-5.66), 0.11 \\
\hline ESRD & $0.29(0.27-0.32)$ & $0.50(0.47-0.53)$ & 1 (Reference) & $1.87(0.67-5.26), 0.23$ \\
\hline Renal transplant & $0.03(0.02-0.04)$ & $0.14(0.12-0.15)$ & 1 (Reference) & $4.92(0.30-81.02), 0.27$ \\
\hline \multicolumn{5}{|l|}{ Secondary outcome } \\
\hline Death & $0.52(0.49-0.56)$ & $0.27(0.25-0.29)$ & 1 (Reference) & $0.54(0.20-1.51), 0.24$ \\
\hline \multicolumn{5}{|l|}{ Safety } \\
\hline Hyperkalemia & $1.03(0.99-1.08)$ & $1.17(1.12-1.21)$ & 1 (Reference) & $1.17(0.65-2.12), 0.59$ \\
\hline
\end{tabular}




\section{Kidney Blood Pressure Research}

Table 3. Incidence Rates and Hazard Ratios for Effectiveness and Safety Outcomes among En al a pril and Selected ACEI Users

Table 4. Incidence Rates and Hazard Ratios for Effectiveness and Safety Outcomes among Valsartan and Selected ARB Users

\begin{tabular}{|c|c|c|c|c|}
\hline & \multicolumn{2}{|c|}{$\begin{array}{c}\text { Incidence Rate per } 100 \text { Patient-years } \\
(95 \% \mathrm{CI})\end{array}$} & \multicolumn{2}{|c|}{ Hazard Ratio (95\% CI), $p$-Value } \\
\hline & Enalapril Users & Selected ACEI Users & Enalapril Users & Selected ACEI Users \\
\hline \multicolumn{5}{|l|}{ Captopril Users } \\
\hline \multicolumn{5}{|l|}{ ITT } \\
\hline \multicolumn{5}{|l|}{ Effectiveness } \\
\hline $\begin{array}{l}\text { Primary composite } \\
\text { outcomes }\end{array}$ & $1.50(1.47-1.54)$ & $2.14(2.09-2.18)$ & 1 (Reference) & $1.42(1.05-1.93), 0.02^{*}$ \\
\hline ESRD & $1.50(1.47-1.54)$ & $2.09(2.05-2.14)$ & 1 (Reference) & 1.39 (1.03-1.89), 0.03* \\
\hline Renal transplant & $0.00(0.00-0.00)$ & $0.04(0.04-0.05)$ & 1 (Reference) & \\
\hline \multicolumn{5}{|l|}{ Secondary outcome } \\
\hline Death & $3.39(3.33-3.44)$ & $4.29(4.23-4.35)$ & 1 (Reference) & $1.25(1.01-1.55), 0.04^{*}$ \\
\hline \multicolumn{5}{|l|}{ Safety } \\
\hline Hyperkalemia & $2.17(2.13-2.21)$ & $2.57(2.52-2.61)$ & 1 (Reference) & $1.19(0.92-1.56), 0.19$ \\
\hline \multicolumn{5}{|l|}{ Fosinopril Users } \\
\hline \multicolumn{5}{|l|}{$\begin{array}{ll}\text { ITT } \\
\end{array}$} \\
\hline \multicolumn{5}{|l|}{ Effectiveness } \\
\hline $\begin{array}{l}\text { Primary composite } \\
\text { outcomes }\end{array}$ & $1.71(1.66-1.76)$ & $2.44(2.38-2.51)$ & 1 (Reference) & $1.42(0.95-2.12), 0.08$ \\
\hline ESRD & $1.71(1.66-1.76)$ & $2.44(2.38-2.51)$ & 1 (Reference) & $1.42(0.95-2.12), 0.08$ \\
\hline Renal transplant & $0.00(0.00-0.00)$ & $0.00(0.00-0.00)$ & & \\
\hline \multicolumn{5}{|l|}{ Secondary outcome } \\
\hline Death & $3.26(3.18-3.33)$ & $3.49(3.42-3.57)$ & 1 (Reference) & $1.07(0.78-1.46), 0.68$ \\
\hline \multicolumn{5}{|l|}{ Safety } \\
\hline Hyperkalemia & $2.36(2.29-2.42)$ & $3.07(3.00-3.15)$ & 1 (Reference) & $1.31(0.92-1.86), 0.14$ \\
\hline \multicolumn{5}{|l|}{ Perindopril Users } \\
\hline \multicolumn{5}{|l|}{ ITT } \\
\hline \multicolumn{5}{|l|}{ Effectiveness } \\
\hline $\begin{array}{l}\text { Primary composite } \\
\text { outcomes }\end{array}$ & $1.56(1.51-1.60)$ & $1.73(1.68-1.77)$ & 1 (Reference) & $1.11(0.74-1.65), 0.61$ \\
\hline ESRD & $1.56(1.51-1.60)$ & $1.62(1.58-1.67)$ & 1 (Reference) & $1.04(0.70-1.57), 0.84$ \\
\hline Renal transplant & $0.00(0.00-0.00)$ & $0.10(0.09-0.11)$ & 1 (Reference) & - \\
\hline \multicolumn{5}{|l|}{ Secondary outcome } \\
\hline Death & $2.85(2.79-2.91)$ & $3.31(3.24-3.37)$ & 1 (Reference) & 1.14 (0.85-1.54), 0.38 \\
\hline \multicolumn{5}{|l|}{ Safety } \\
\hline Hyperkalemia & $2.18(2.13-2.24)$ & $2.238(2.18-2.29)$ & 1 (Reference) & $1.04(0.73-1.48), 0.82$ \\
\hline
\end{tabular}

*statistically significant. ACEIs: angiotensin-converting enzyme inhibitors; ITT: intention-to-treat analysis; CI: confidence interval; ESRD: end-stage renal disease

\begin{tabular}{|c|c|c|c|c|}
\hline & \multicolumn{2}{|c|}{$\begin{array}{c}\text { Incidence Rate per } 100 \text { Patient-years } \\
(95 \% \mathrm{CI})\end{array}$} & \multicolumn{2}{|c|}{ Hazard Ratio (95\% CI), $p$-Value } \\
\hline & Valsartan Users & Selected ARB Users & Valsartan Users & Selected ARB Users \\
\hline \multicolumn{5}{|l|}{ Irbesartan } \\
\hline \multicolumn{5}{|l|}{$\begin{array}{l}\text { ITT } \\
\end{array}$} \\
\hline \multicolumn{5}{|l|}{ Effectiveness } \\
\hline Primary composite outcomes & $2.51(2.46-2.56)$ & $3.37(3.31-3.43)$ & 1 (Reference) & 1.35 (1.03-1.78), 0.03* \\
\hline ESRD & $2.47(2.42-2.52)$ & $3.29(3.23-3.35)$ & 1 (Reference) & 1.34 (1.01-1.77), $0.04^{*}$ \\
\hline Renal transplant & $0.04(0.03-0.05)$ & $0.08(0.07-0.09)$ & 1 (Reference) & $2.15(0.29-16.30), 0.46$ \\
\hline \multicolumn{5}{|l|}{ Secondary outcome } \\
\hline Death & $3.98(3.92-4.05)$ & $3.71(3.65-3.77)$ & 1 (Reference) & 0.95 (0.75-1.21), 0.67 \\
\hline \multicolumn{5}{|l|}{ Safety } \\
\hline Hyperkalemia & $3.27(3.21-3.33)$ & $3.24(3.18-3.30)$ & 1 (Reference) & $1.00(0.77-1.30), 0.98$ \\
\hline \multicolumn{5}{|l|}{ Losartan } \\
\hline \multicolumn{5}{|l|}{ ITT } \\
\hline \multicolumn{5}{|l|}{ Effectiveness } \\
\hline Primary composite outcomes & $2.31(2.27-2.35)$ & $2.64(2.60-2.69)$ & 1 (Reference) & 1.15 (0.89-1.48), 0.29 \\
\hline ESRD & $2.26(2.22-2.30)$ & $2.49(2.45-2.54)$ & 1 (Reference) & $1.10(0.85-1.43), 0.45$ \\
\hline Renal transplant & $0.05(0.04-0.05)$ & $0.15(0.14-0.16)$ & 1 (Reference) & $3.10(0.71-13.44), 0.13$ \\
\hline \multicolumn{5}{|l|}{ Secondary outcome } \\
\hline Death & $3.62(3.57-3.68)$ & $3.67(3.62-3.73)$ & 1 (Reference) & $1.03(0.84-1.28), 0.75$ \\
\hline \multicolumn{5}{|l|}{ Safety } \\
\hline Hyperkalemia & $3.06(3.01-3.11)$ & $3.40(3.34-3.45)$ & 1 (Reference) & $1.10(0.88-1.37), 0.42$ \\
\hline
\end{tabular}

*statistically significant. ARBs: angiotensin II receptor blockers; ITT: intention-to-treat analysis; CI: confidence interval; ESRD: end-stage renal disease 


\section{Kidney Blood Pressure Research}

Table 5. Incidence Rates and Hazard Ratios for Effectiveness and Safety Outcomes among ACEI and ARB Users Aged 65 Years or Older

\begin{tabular}{|c|c|c|c|c|}
\hline & \multicolumn{2}{|c|}{$\begin{array}{c}\text { Incidence Rate per } 100 \text { Patient-years } \\
(95 \% \mathrm{CI})\end{array}$} & \multicolumn{2}{|c|}{ Hazard Ratio (95\% CI), $p$-Value } \\
\hline & ACEI Users & ARB Users & ACEI Users & ARB Users \\
\hline \multicolumn{5}{|l|}{ ITT } \\
\hline \multicolumn{5}{|l|}{ Effectiveness } \\
\hline $\begin{array}{l}\text { Primary composite } \\
\text { outcomes }\end{array}$ & $1.38(1.35-1.40)$ & $2.53(2.49-2.57)$ & 1 (Reference) & $1.84(1.44-2.33),<0.01^{*}$ \\
\hline ESRD & $1.35(1.33-1.38)$ & $2.39(2.35-2.43)$ & 1 (Reference) & $1.77(1.39-2.26),<0.01^{*}$ \\
\hline Renal transplant & $0.02(0.02-0.03)$ & $0.14(0.13-0.15)$ & 1 (Reference) & 5.14 (1.12-23.61), $0.04^{*}$ \\
\hline \multicolumn{5}{|l|}{ Secondary outcome } \\
\hline Death & $6.12(6.06-6.18)$ & $5.98(5.91-6.04)$ & 1 (Reference) & $1.01(0.88-1.16), 0.91$ \\
\hline \multicolumn{5}{|l|}{ Safety } \\
\hline Hyperkalemia & $3.12(3.38-3.46)$ & $4.40(4.35-4.46)$ & 1 (Reference) & 1.25 (1.05-1.49), $0.01^{*}$ \\
\hline \multicolumn{5}{|l|}{$\mathrm{AT}$} \\
\hline \multicolumn{5}{|l|}{ Effectiveness } \\
\hline $\begin{array}{l}\text { Primary composite } \\
\text { outcomes }\end{array}$ & $0.32(0.27-0.37)$ & $1.32(1.23-1.41)$ & 1 (Reference) & 4.25 (0.80-22.61), 0.09 \\
\hline ESRD & $0.22(0.18-0.26)$ & $0.82(0.76-0.90)$ & 1 (Reference) & $3.72(0.49-28.42), 0.20$ \\
\hline Renal transplant & $0.10(0.08-0.13)$ & $0.49(0.44-0.55)$ & 1 (Reference) & $5.44(0.29-103.11), 0.26$ \\
\hline \multicolumn{5}{|l|}{ Secondary outcome } \\
\hline Death & $1.36(1.26-1.46)$ & $0.33(0.28-0.37)$ & 1 (Reference) & 0.26 (0.05-1.28), 0.10 \\
\hline \multicolumn{5}{|l|}{ Safety } \\
\hline Hyperkalemia & $2.17(2.05-2.30)$ & $1.80(1.70-1.91)$ & 1 (Reference) & $00.82(0.36-1.89), 0.65$ \\
\hline $\begin{array}{l}{ }^{*} \text { statistically significa } \\
\text { blockers; ITT: intent } \\
\text { renal disease }\end{array}$ & ACEIs: angiotensi & converting enzyn & hibitors; ARB & $\begin{array}{l}\text { angiotensin II receptor } \\
\text { interval; ESRD: end-stage }\end{array}$ \\
\hline
\end{tabular}

outcome (HR: 1.77, 95\% CI: 1.01-3.10) in the ITT analysis (data not shown). No significant results were found in the analysis for ARBs (data not shown).

\section{Discussion}

The present study utilized a real-world cohort to assess the renal outcomes, mortality, and safety of ACEIs and ARBs among diabetic patients with proteinuria. To the best of our knowledge, this is the first study assessing the renoprotective effects of individual ACEIs and ARBs in diabetic patients with proteinuria. The findings of this study suggest that ACEI users have a lower risk of ESRD compared to ARB users, which is consistent with a prior study conducted in Taiwan [9]. Although not statistically significant, ACEIs were systematically shown to be superior to ARBs regarding the dialysis and mortality outcomes in patients with diabetes in a systematic review [26]. Conversely, another systematic review demonstrated no significant differences between ACEIs and ARBs with respect to kidney protection in patients with albuminuria and other cardiovascular risk factors, such as diabetes, hypertension and hyperlipidemia [27]. However, the proportion of diabetic patients in that systematic review was low. Finally, a recent network meta-analysis comparing the effects of antihypertensive agents in diabetic patients with kidney disease showed that the ACEI-CCB combination therapy of fosinopril and amlodipine appeared to be the most efficacious in reducing proteinuria [28]. While the ACEI-ARB combination was not recommended due to safety concerns $[6,7]$, based on the results of the network meta-analysis, a combination of ACEIs or ARBs with other antihypertensive agents may deserve further investigation.

Regarding the safety profile, our study revealed that ACEI users have a lower risk of hyperkalemia compared to ARB users, and the result was different from a previous clinical trial which reported that ARB therapy was less likely to increase serum potassium levels compared to ACEI therapy among people with renal failure [29]. There are some potential explanations for such different findings between studies. First, the number of participants in 


\section{Kidney Blood Pressure Research}

Kidney Blood Press Res 2017;42:358-368

\begin{tabular}{l|l}
\hline DOI: $10.1159 / 000477946$ & (c) 2017 The Author(s). Published by S. Karger AG, Basel
\end{tabular}

Published online: June 13, 2017

www.karger.com/kb

Hsu et al.: Renoprotective Effect of ACEIs and ARBs

the clinical trial was relatively small $(n=35)$ [29], which may not be generalizable to a diverse population. Second, the clinical trial only compared lisinopril and valsartan, and these two drugs may not completely represent the medication categories they belong to.

In order to explain why ACEIs have more renal benefit and safety than ARBs, the mechanisms of action should be taken into consideration. First, angiotensin II is believed to cause renal injury initially via its ability to cause ischemia and local inflammation [30, 31]. However, subsequent studies have found that angiotensin II may be renal protective and beneficial by potentiating the myogenic responses to pressure changes [32, 33]. Although ACEIs block the enzyme that converts angiotensin I to angiotensin II systemically, angiotensin II can still be generated in the periphery, such as the brain and kidneys [34, 35]. In addition to angiotensin-converting enzyme (ACE), there are some alternative enzymes that could be responsible for generating the angiotensin II, such as chymase serine proteases [36]. Therefore, angiotensin II can still be generated and maintained at a normal level when ACEIs are administrated.

In addition, the blockage of the angiotensin II type 1 (AT1) receptor by ARBs may stimulate the angiotensin II type 2 (AT2) receptor. The activation of the AT2 receptor causes vasodilation, which improves blood pressure control. Nonetheless, the activated AT2 receptor also mediates programmed cell death, namely apoptosis $[37,38]$. In other word, whether the excessive activation of the AT2 receptor in the human body is good or bad remains controversial [37-41]. Unlike ARBs, ACEIs act as a blocker upstream to the renin-angiotensin system (RAS), and have no direct effect on AT2 receptors. Lastly, ACEIs attenuated renal fibrosis, possibly by suppressing the mast cell degranulation, in rats with unilateral ureteral obstruction $[42,43]$. Given that currently there are no clear mechanisms that may explain the superior protective effect of ACEIs compared to ARBs, further experimental and human studies are warranted.

In the analyses of individual ACEIs, captopril had a higher risk of ESRD and mortality compared to enalapril. Given that the captopril is a short-acting ACEI, it is possible that physicians may have different prescribing patterns or considerations when using captopril versus other ACEIs. Patients treated with captopril have been reported to be the most underdosed among all ACEI users, and therefore might not be able to achieve full treatment benefits [44]. In addition, captopril could be prescribed to control acute or chronic hypertension, but it is not possible to differentiate these two types of use or adjust for patients' actual blood pressure control in a claim-based study. The weaker effect of captopril on the long-term outcomes associated with ESRD and death observed in the present study could be due to the shorter duration of action, more frequent underdosage, or insufficient control of unobserved confounders for the different prescribing patterns and patient populations. In the analyses of individual ARBs, irbesartan was associated with a higher risk of ESRD compared to valsartan, but the exact mechanisms that caused the poorer outcomes were unclear. As expected, we did not find any differences in the risk of hyperkalemia among individual ACEI and ARB medications, since both ACEIs and ARBs can inhibit the reninangiotensin system and raise the serum potassium level in patients with renal insufficiency or diabetes [45]. However, it is worth noting that the small sample size of the selected ACEI and ARB medications could have reduced our power to detect the differences.

In the pre-specified subgroup analysis of diabetic patients who were 65 years of age or older, the ARB users had higher rates of ESRD and hyperkalemia than the ACEI users, and such an effect was more profound than in the overall adult patient population. Consistent with the findings in the adult sample, when comparing individual ACEIs, we found that the elderly patients with captopril had an increased risk of the composite adverse renal outcome of ESRD and renal transplantation compared to those on enalapril. No significant results were found in the analyses comparing individual ARBs. Elderly patients with kidney diseases are particularly sensitive to the risk of adverse events, but, on the whole, the incidence of hyperkalemia events was not significantly different among individual ACEI 


\section{Kidney Blood Pressure Research}

and ARB medications in the present study. Furthermore, our study did not demonstrate any significant differences in the mortality among individual ACEIs and ARBs.

The present study was notable for its representativeness of having a nationwide cohort, but it was still subject to several limitations. First, some health-related information is not available in the claims database, such as lab data (e.g., the amount of proteinuria, creatinine level) and health-related behaviors. Therefore, we were unable to stratify our analysis by the severity of proteinuria. Second, given that we did not compare individual ACEIs with individual ARBs, we cannot draw a conclusion that all ACEIs are better than ARBs. Third, we only excluded patients with more than 12 dialysis sessions within 180 days prior to the index date, which may still leave patients with renal impairment in our sample. However, our sensitivity analysis showed that the results stayed similar with the main analysis even we excluded patients with at least one dialysis session (HR for the primary composite outcomes: 1.31 [1.15-1.50], $\mathrm{p}<0.01$; HR for death: 1.09 [0.98-1.22], $\mathrm{p}=0.11$; HR for the safety outcome: 1.07 [0.97-1.19], $\mathrm{p}=0.19$ ). In addition, we controlled the baseline renal diseases in our analysis, which should also help to reduce the bias. Finally, the sample size for the elderly could have been too small to detect differences in some clinical outcomes, such as the renal transplant event. Future studies with greater samples are warranted.

\section{Conclusion}

Our findings support that ACEIs are relatively renoprotective and safe treatments as compared with ARBs in diabetic patients with proteinuria. Among the commonly used ACEI drugs, enalapril was a superior treatment option than captopril in preventing kidney failure and reducing the risk of mortality. On the other hand, irbesartan appeared to be less renoprotective than valsartan. In older diabetic patients with proteinuria, ACEIs were also a better choice compared to ARBs. Captopril showed a higher risk of ESRD and renal transplantation than enalapril, and all the selected ARBs (irbesartan, losartan, and valsartan) performed similarly in the elderly population. Although the analysis of hyperkalemia showed no significant differences among individual ACEI and ARB agents, physicians should still pay attention to the potential hyperkalemia effect when using these drugs.

\section{Disclosure Statement}

This study was partially funded by a research grant from the Ministry of Science and Technology in Taiwan (MOST 104-2314-B-002-095). The funders had no role in the study design, data collection and analysis, result interpretation, publication decision, or manuscript preparation.

\section{Acknowledgements}

The study is based on data from the National Health Insurance Research Database provided by the National Health Insurance Administration, Ministry of Health and Welfare and managed by the National Health Research Institutes. The interpretation and conclusions contained herein do not represent those of the National Health Insurance Administration, Ministry of Health and Welfare, or National Health Research Institutes.

\section{References}

1 U.S. Renal Data System: USRDS 2012 Annual Data Report: Atlas of chronic kidney disease and end-stage renal disease in the United States, 2012. 


\section{Kidney \\ Blood Pressure Research}

- Ganesh J, Viswanathan V: Management of diabetic hypertensives. Indian J Endocrinol Metab 2011;15:S374379.

- 3 Palmer SC, Mavridis D, Navarese E, Craig JC, Tonelli M, Salanti G, Wiebe N, Ruospo M, Wheeler DC, Strippoli GFM: Comparative efficacy and safety of blood pressure-lowering agents in adults with diabetes and kidney disease: a network meta-analysis. Lancet 2015;385:2047-2056.

4 Gu J, Yang M, Qi N, Mei S, Chen J, Song S, Jing Y, Chen M, He L, Sun L, Hu H, Li L, Wuthrich RP, Wu M, Mei C: Olmesartan Prevents Microalbuminuria in db/db Diabetic Mice Through Inhibition of Angiotensin II/p38/ SIRT1-Induced Podocyte Apoptosis. Kidney Blood Press Res 2016;41:848-864.

5 American Diabetes Association: Standards of medical care in diabetes 2015, 2015.

-6 Fried LF, Emanuele N, Zhang JH, Brophy M, Conner TA, Duckworth W, Leehey DJ, McCullough PA, O'Connor T, Palevsky PM, Reilly RF, Seliger SL, Warren SR, Watnick S, Peduzzi P, Guarino P: Combined angiotensin inhibition for the treatment of diabetic nephropathy. N Engl J Med 2013;369:1892-1903.

7 Fernandez Juarez G, Luno J, Barrio V, de Vinuesa SG, Praga M, Goicoechea M, Cachofeiro V, Nieto J, Fernandez Vega F, Tato A, Gutierrez E: Effect of dual blockade of the renin-angiotensin system on the progression of type 2 diabetic nephropathy: a randomized trial. Am J Kidney Dis 2013;61:211-218.

-8 Hsu TW, Liu JS, Hung SC, Kuo KL, Chang YK, Chen YC, Hsu CC, Tarng DC: Renoprotective effect of reninangiotensin-aldosterone system blockade in patients with predialysis advanced chronic kidney disease, hypertension, and anemia. JAMA Intern Med 2014;174:347-354.

-9 Wu LS, Chang SH, Chang GJ, Liu JR, Chan YH, Lee HF, Wen MS, Chen WJ, Yeh YH, Kuo CT, See LC: A comparison between angiotensin converting enzyme inhibitors and angiotensin receptor blockers on end stage renal disease and major adverse cardiovascular events in diabetic patients: a population-based dynamic cohort study in Taiwan. Cardiovasc Diabetol 2016;15:56.

10 Wooten JM: Pharmacotherapy considerations in elderly adults. South Med J 2012;105:437-445.

$\checkmark 11$ Mangoni AA, Jackson SH: Age-related changes in pharmacokinetics and pharmacodynamics: basic principles and practical applications. Br J Clin Pharmacol 2004;57:6-14.

-12 Crooks J, O'Malley K, Stevenson IH: Pharmacokinetics in the elderly. Clin Pharmacokinet 1976;1:280-296. Klotz U: Pharmacokinetics and drug metabolism in the elderly. Drug Metab Rev 2009;41:67-76. Cheng TM: Taiwan's new national health insurance program: genesis and experience so far. Health Aff (Millwood) 2003;22:61-76.

15 Cheng P, Neugaard B, Foulis P, Conlin PR: Hemoglobin A1c as a Predictor of Incident Diabetes. Diabetes Care 2011;34:610-615.

16 Shireman TI, Mahnken JD, Phadnis MA, Ellerbeck EF, Wetmore JB: Comparative Effectiveness of ReninAngiotensin System Antagonists in Maintenance Dialysis Patients. Kidney Blood Press Res 2016;41:873885.

17 Malik U, Raizada V: Some Aspects of the Renin-Angiotensin-System in Hemodialysis Patients. Kidney Blood Press Res 2015;40:614-622.

18 National health insurance administration ministry of health and welfare: Amount of drug use analysis, 2014.

19 WHO Collaborating Centre for Drug Statistics Methodology: ATC, 2015.

$>20$ Chang G-M, Tung Y-C: Factors Associated with Pneumonia Outcomes: A Nationwide Population-Based Study over the 1997-2008 Period. J Gen Intern Med 2012;27:527-533.

21 Naughton CA: Drug-induced nephrotoxicity. Am Fam Physician 2008;78:743-750.

-22 Lazarus B, Chen Y, Wilson FP, Sang Y, Chang AR, Coresh J, Grams ME: Proton Pump Inhibitor Use and the Risk of Chronic Kidney Disease. JAMA Intern Med 2016;176:238-246.

23 Morales E, Caro J, Gutierrez E, Sevillano A, Aunon P, Fernandez C, Praga M: Diverse diuretics regimens differentially enhance the antialbuminuric effect of renin-angiotensin blockers in patients with chronic kidney disease. Kidney Int 2015;88:1434-1441.

-24 Normand S-LT, Landrum MB, Guadagnoli E, Ayanian JZ, Ryan TJ, Cleary PD, McNeil BJ: Validating recommendations for coronary angiography following acute myocardial infarction in the elderly: A matched analysis using propensity scores. J Clin Epidemiol 2001;54:387-398.

-25 Austin PC: The use of propensity score methods with survival or time-to-event outcomes: reporting measures of effect similar to those used in randomized experiments. Stat Med 2014;33:1242-1258. 


\section{Kidney \\ Blood Pressure Research}

-26 Wu HY, Huang JW, Lin HJ, Liao WC, Peng YS, Hung KY, Wu KD, Tu YK, Chien KL: Comparative effectiveness of renin-angiotensin system blockers and other antihypertensive drugs in patients with diabetes: systematic review and bayesian network meta-analysis. BMJ 2013;347:f6008.

-27 Maione A, Navaneethan SD, Graziano G, Mitchell R, Johnson D, Mann JF, Gao P, Craig JC, Tognoni G, Perkovic V, Nicolucci A, De Cosmo S, Sasso A, Lamacchia O, Cignarelli M, Manfreda VM, Gentile G, Strippoli GF: Angiotensin-converting enzyme inhibitors, angiotensin receptor blockers and combined therapy in patients with micro- and macroalbuminuria and other cardiovascular risk factors: a systematic review of randomized controlled trials. Nephrol Dial Transplant 2011;26:2827-2847.

-28 Huang R, Feng Y, Wang Y, Qin X, Melgiri ND, Sun Y, Li X: Comparative Efficacy and Safety of Antihypertensive Agents for Adult Diabetic Patients with Microalbuminuric Kidney Disease: A Network Meta-Analysis. PLoS One 2017;12:e0168582.

29 Bakris GL, Siomos M, Richardson D, Janssen I, Bolton WK, Hebert L, Agarwal R, Catanzaro D: ACE inhibition or angiotensin receptor blockade: Impact on potassium in renal failure. Kidney Int 2000;58:2084-2092.

-30 Long DA, Price KL, Herrera-Acosta J, Johnson RJ: How does angiotensin II cause renal injury? Hypertension 2004;43:722-723.

31 Cao W, Jin L, Zhou Z, Yang M, Wu C, Wu L, Cui S: Overexpression of Intrarenal Renin-Angiotensin System in Human Acute Tubular Necrosis. Kidney Blood Press Res 2016;41:746-756.

32 Benndorf RA, Krebs C, Hirsch-Hoffmann B, Schwedhelm E, Cieslar G, Schmidt-Haupt R, Steinmetz OM, Meyer-Schwesinger C, Thaiss F, Haddad M, Fehr S, Heilmann A, Helmchen U, Hein L, Ehmke H, Stahl RA, Boger RH, Wenzel UO: Angiotensin II type 2 receptor deficiency aggravates renal injury and reduces survival in chronic kidney disease in mice. Kidney Int 2009;75:1039-1049.

33 Griffin KA, Bidani AK: Angiotensin II type 2 receptor in chronic kidney disease: the good side of angiotensin II? Kidney Int 2009;75:1006-1008.

-34 Wolf G, Butzmann U, Wenzel UO: The renin-angiotensin system and progression of renal disease: from hemodynamics to cell biology. Nephron Physiol 2003;93:P3-13.

35 Imig JD, Navar GL, Zou L-X, O’Reilly KC, Allen PL, Kaysen JH, Hammond TG, Navar LG: Renal endosomes contain angiotensin peptides, converting enzyme, and AT1A receptors. Am J Physiol Renal Physiol 1999;277:F303-F311.

-36 Arakawa K: Serine protease angiotensin II systems. J Hypertens Suppl 1996;14:S3-7.

37 Ardaillou R: Angiotensin II receptors. J Am Soc Nephrol 1999;10:S30-39.

38 Chung 0, Kuhl H, Stoll M, Unger T: Physiological and pharmacological implications of AT1 versus AT2 receptors. Kidney Int Suppl 1998;67:S95-99.

39 Levy BI: How to explain the differences between renin angiotensin system modulators. Am J Hypertens 2005;18:134s-141s.

$\$ 40$ Reudelhuber TL: The continuing saga of the AT2 receptor: a case of the good, the bad, and the innocuous. Hypertension 2005;46:1261-1262.

-41 Levy BI: Can angiotensin II type 2 receptors have deleterious effects in cardiovascular disease? Implications for therapeutic blockade of the renin-angiotensin system. Circulation 2004;109:8-13.

-42 Liu Y: Renal fibrosis: new insights into the pathogenesis and therapeutics. Kidney Int 2006;69:213-217.

-43 Sun N, Zhai L, Li H, Shi LH, Yao Z, Zhang B: Angiotensin-Converting Enzyme Inhibitor (ACEI)-Mediated Amelioration in Renal Fibrosis Involves Suppression of Mast Cell Degranulation. Kidney Blood Press Res 2016;41:108-118.

44 Hansen ML, Gislason GH, Kober L, Schramm TK, Folke F, Buch P, Abildstrom SZ, Madsen M, Rasmussen S, Torp-Pedersen C: Different angiotensin-converting enzyme inhibitors have similar clinical efficacy after myocardial infarction. Br J Clin Pharmacol 2008;65:217-223.

45 Preston RA, Hirsh MM, Oster MD, Jr., Oster HM: University of Miami Division of Clinical Pharmacology therapeutic rounds: drug-induced hyperkalemia. Am J Ther 1998;5:125-132. 